\title{
ESTUDO DA SÍNTESE DA ZEÓLITA MCM-71
}

\section{Caroline Luvison, Marcelo L. Mignoni e Sibele B. C. Pergher*}

Departamento de Química, Universidade Regional Integrada do Alto Uruguai e das Missões, Campus Erechim, Av. Sete de Setembro, 1621, 99700-000 Erechim - RS, Brasil

Recebido em 21/11/07; aceito em 24/7/08; publicado na web em 15/12/08

\begin{abstract}
STUDY OF MCM-71 ZEOLITE SYNTHESIS. A new kind of material, denominated MCM-71, was synthesized and characterized by several complementary techniques: X Ray Diffractometry, textural analysis by nitrogen adsorption, Scanning electronic microscopy and infrared spectroscopy. MCM-71 zeolite was successfully synthesized by hydrothermal synthesis in the presence of triethanolamine. Mordenite phase as impurity was not detected, otherwise quartz was observed. The MCM-71 sample obtained presented a BET surface area of $20 \mathrm{~m}^{2} / \mathrm{g}$ in the as synthesized form and of $85 \mathrm{~m}^{2} / \mathrm{g}$ in protonic form. By SEM was observed crystals with rectangular shape with average size of $2 \times 0,2 \times 0,05 \mu \mathrm{m}$ and this crystals were agglomerated in spherical particles with average diameter between 14 and $24 \mu \mathrm{m}$.
\end{abstract}

Keywords: MCM-71; zeolites; synthesis.

\section{INTRODUÇÃO}

Zeólitas são compostos microporosos que apresentam um papel importante em vários áreas tecnológicas, principalmente como catalisadores. ${ }^{1,2}$ As razões de seu êxito em catálise são sua alta área superficial, capacidade de adsorção, seus centros ácidos, tamanhos de seus canais e cavidades e sua seletividade de forma. Estas características fazem com que as zeólitas sejam materiais interessantes para serem utilizados também como trocadores iônicos, peneiras moleculares e adsorventes. Neste sentido é interessante sempre buscar novos materiais zeolíticos ainda pouco estudados.

A síntese da zeólita MCM-71 foi descrita recentemente. ${ }^{3,4}$ Esse novo material possui um sistema de canais tridimensionais retos, de abertura elíptica, formados por $10 \operatorname{MR}(0,65$ x 0,43 nm) que são intersectados por outro sistema de canais senozoidais definidos por abertura de 8 MR (0,47 x 0,36 nm), Figura 1 .

Este material foi sintetizado com uma relação $\mathrm{Si} / \mathrm{Al} \sim 8$, que faz que seja um catalisador interessante para reações ácido-base. Zeólitas de poros médios têm sido empregadas em reações que requerem seletividade de forma, tais como adição de amina no metanol para produzir monometilamina pela inibição da formação de di- e trimetilamine; em reações de isomerização de n-alcanos de frações leves de nafta para aumento da octanagem e, em reações de reforma catalítica do diesel pela remoção seletiva de parafinas de cadeias lineares para aumentar o índice de viscosidade do produto. ${ }^{5}$

O objetivo deste trabalho foi estudar a síntese e caracterização da zeólita MCM-71.

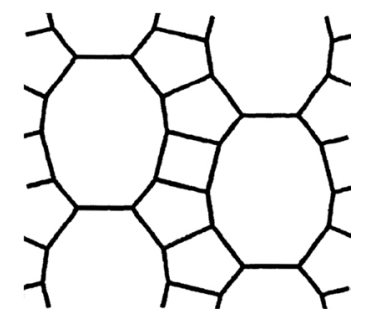

Figura 1. Esquema dos canais elípticos de 10MR

*e-mail: pergher@uri.com.br

\section{PARTE EXPERIMENTAL}

\section{Síntese da MCM-71}

A síntese da zeólita MCM-71 foi realizada seguindo o procedimento descrito na patente (US Pat. 6,645,462 e WO 03/097531) ) $^{3,4}$ empregando os seguintes reagentes: sílica coloidal (30\%), $\mathrm{Al}(\mathrm{OH})_{3}, \mathrm{KOH}$, trietanolamina $(\mathrm{R})$ e $\mathrm{H}_{2} \mathrm{O}$. O gel formado, possuindo a composição molar $\mathrm{Si} / \mathrm{Al}_{2}=21 ; \mathrm{H}_{2} \mathrm{O} / \mathrm{Si}=30 ; \mathrm{OH} / \mathrm{Si}=0,375 ; \mathrm{K}^{+} / \mathrm{Si}=0,375 ; \mathrm{R} / \mathrm{Si}$ $=0,20$, foi transferido para autoclaves de aço inoxidável com cobertura interna de Teflon e mantido a $160{ }^{\circ} \mathrm{C}$ por 15 dias e $180{ }^{\circ} \mathrm{C}$ por mais 5 dias. Após este período, o material foi separado por centrifugação e lavado até que a água de lavagem tivesse um pH inferior a 9,0.

A forma protonada do material, $\mathrm{H}^{+}-\mathrm{MCM} 71$, foi preparada por troca catiônica com uma solução $0,1 \mathrm{~mol} / \mathrm{L}$ de $\mathrm{NH}_{4} \mathrm{NO}_{3}$ a $80{ }^{\circ} \mathrm{C}$ por 3 h. A razão zeólita/solução empregada foi de $1 \mathrm{~g}$ de zeólita/100 g de solução. Os materiais preparados foram calcinados a $500{ }^{\circ} \mathrm{C}$ por $3 \mathrm{~h}$ (taxa de aquecimento $3{ }^{\circ} \mathrm{C} / \mathrm{min}$ ).

\section{Caracterização dos materiais}

Os materiais preparados foram caracterizados por diversas técnicas complementares: difração de Raios X, análise textural por adsorção de nitrogênio, microscopia eletrônica de varredura e espectroscopia no infravermelho.

As análises de difração de raios $\mathrm{X}$ foram realizadas num difraktometer modelo D5000 (Siemens) utilizando filtro de Ni e radiação $\mathrm{Cu}-\mathrm{k} \alpha(\lambda=1,54 \AA)$.

A caracterização textural dos materiais foi realizada utilizando um autosorb-1 da Quantachrome (Nova-2200e). Antes da análise, cerca de $100 \mathrm{mg}$ de amostra foram tratadas a vácuo, a uma temperatura de $300^{\circ} \mathrm{C}$ por $3 \mathrm{~h}$. As medidas foram realizadas na temperatura do $\mathrm{N}_{2}$ líquido.

As análises de microscopia eletrônica de varredura foram realizadas em um microscópio MEV SSZ 550 Shimadzu e as amostras foram previamente recobertas com um filme de ouro.

A análise de espectrocopia no infravermelho foi realizada em um espectrofotômetro FTIR Shimadzu, modelo 8300. O espectro foi obtido com 32 varreduras e com $4 \mathrm{~cm}^{-1}$ de resolução. Para a análise, a amostra foi preparada na forma de pastilhas de aproximadamente 
$2 \mathrm{~mm}$ de espessura e $5 \mathrm{~mm}$ de diâmetro. Uma diluição de cerca de $10 \%$ de amostra foi realizada com $\mathrm{KBr}$.

\section{RESULTADOS E DISCUSSÃO}

As análises de difração de raios X mostraram que em 15 dias o material obtido era amorfo e que em 20 dias o material já apresentava cristalinidade e um difratograma típico da MCM-71. ${ }^{3,4}$ A Tabela 1 apresenta a comparação do material sintetizado com o da literatura. ${ }^{3,4}$ Observa-se boa concordância entre os sinais. Não se observa a fase mordenita $(2 \theta=6,5 ; 8,6 ; 9,7 ; 13,4 ; 13,8 ; 19,6 ; 22,2 ; 25,6 ; 26,2$; $27,6)$ como impureza que, segundo a literatura, pode estar presente. ${ }^{3}$ Entretanto, nota-se a presença de quartzo $\left(2 \theta \sim 27^{\circ}\right)$.

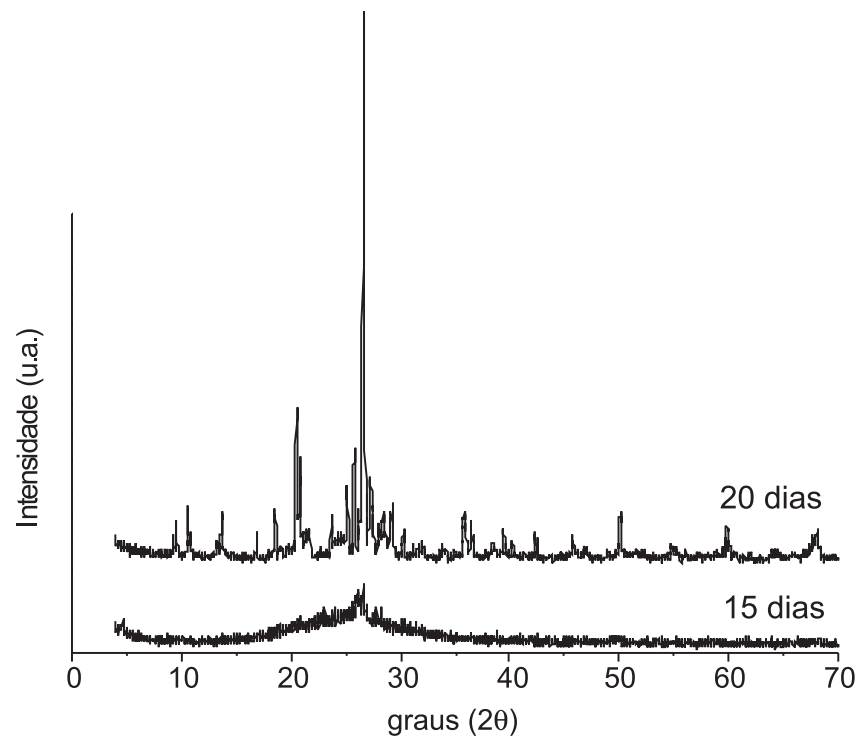

Figura 2. Difratograma das amostras preparadas em 15 e 20 dias

As isotermas de adsorção/dessorção de $\mathrm{N}_{2}$ das amostras recémsintetizada e da amostra que sofreu troca iônica com $\mathrm{H}^{+}$estão representadas na Figura 3. No caso da MCM-71 recém-sintetizada, que possui íons $\mathrm{K}^{+}$como compensadores de carga, obteve-se baixa adsorção de $\mathrm{N}_{2}$ indicando uma baixa acessibilidade desta molécula à superfície interna da estrutura zeolítica. Este fato ocorre também com outras estruturas zeolíticas, como por exemplo, a zeólita A, que na forma recém-sintetizada $\left(\mathrm{Na}^{+}-\mathrm{A}\right)$ não possui acessibilidade às moléculas de $\mathrm{N}_{2}$. Entretanto, quando a zeólita A é trocada com íons $\mathrm{Ca}^{+2}$ a estrutura fica acessível às moléculas de $\mathrm{N}_{2} \cdot{ }^{5,6} \mathrm{Em}$ virtude disso, realizou-se na MCM-71 uma troca catiônica com íons $\mathrm{NH}_{4}^{+}$e através de posterior calcinação a $500{ }^{\circ} \mathrm{C}$ obtive-se o material $\mathrm{H}^{+}$-MCM-71. Este material apresentou um aumento na quantidade de nitrogênio adsorvida ao ser comparado com a MCM-71 recém-sintetizada. Isso ocorre porque a acessibilidade à estrutura interna aumenta com a diminuição do tamanho do cátion compensador de carga.

Através dos dados de adsorção calcularam-se a área superficial específica e os volumes de poros (Tabela 2). Observa-se um aumento na área superficial específica de 20 a $85 \mathrm{~m}^{2} / \mathrm{g}$, devido ao aumento da microporosidade ao realizar-se a troca iônica com $\mathrm{H}^{+}$. Ernst et al. ${ }^{7}$ obtiveram valores de $380 \mathrm{~m}^{2} / \mathrm{g}$ para $\mathrm{H}^{+}$-MCM-71. O baixo valor obtido para área superficial provavelmente se deve ao fato de a troca iônica com $\mathrm{H}^{+}$não ter sido total. Não descartamos a possibilidade do material conter trietanolamina nos poros, que não tenha sido eliminada de forma definitiva. Também devemos levar em consideração a contribuição do quartzo.

As micrografias de MEV (Figura 4) apresentaram a morfologia do material MCM-71, onde se observam cristais retangulares aglomerados
Tabela 1. Comparação dos picos do difratograma da amostra sintetizada com os da literatura

\begin{tabular}{lccc}
\hline Padrão [3,4] & & \multicolumn{2}{c}{ MCM71 (20 dias) } \\
$2 \theta\left(^{\circ}\right)$ & I Rel ${ }^{*}$ & $2 \theta\left(^{\circ}\right)$ & I Rel \\
\hline 9,23 & w-s & 9,24 & w \\
9,51 & w-s & 9,54 & w \\
10,58 & m-s & 10,59 & m \\
13,61 & w-m & 13,60 & m \\
16,83 & w-m & 16,79 & w \\
18,50 & m-s & 18,51 & m \\
21,23 & vw-w & 21,21 & w \\
23,69 & w-m & 23,72 & m \\
25,126 & s-vs & 25,14 & s \\
25,793 & s-vs & 25,79 & vs \\
26,576 & w & 26,03 & $\#$ \\
28,39 & vw-w & 28,38 & w \\
30,261 & vw-w & 30,28 & w \\
48,98 & vw & 48,87 & vw \\
\hline
\end{tabular}

(*) vs = muito forte $(80-100) ; \mathrm{s}=$ forte $(60-80) ; \mathrm{m}=$ médio $(40-60)$; $\mathrm{w}=$ fraco $(20-40) ; \mathrm{w}=$ muito fraco $(0-20) ;(\#)$ ocorre sobreposição de picos com o do quartzo

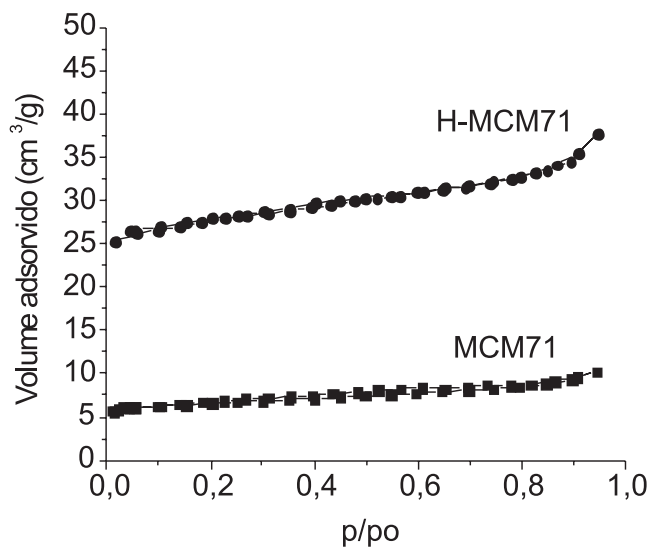

Figura 3. Isotermas de adsorção de $N_{2}$ da MCM-71 e da HMCM-71

Tabela 2. Área superficial específica e volume de poros calculados a partir dos dados de adsorção/dessorção de $\mathrm{N}_{2}$

\begin{tabular}{lccc}
\hline Amostra & $\mathrm{A}_{\mathrm{BET}}\left(\mathrm{m}^{2} / \mathrm{g}\right)$ & $\mathrm{V}_{\text {TOTAL }}\left(\mathrm{cm}^{3} / \mathrm{g}\right)$ & $\mathrm{V}_{\text {Micro }}\left(\mathrm{cm}^{3} / \mathrm{g}\right)$ \\
\hline MCM-71 & 20,64 & 0,016 & 0,007 \\
H-MCM-71 & 85,68 & 0,058 & 0,035 \\
\hline
\end{tabular}

formando esferas. Os aglomerados possuem diâmetro de 14 a 24 m. Os cristais possuem um tamanho médio de $2 \times 0,2 \times 0,05 \mu \mathrm{m}$. Este tamanho é muito inferior ao encontrado por Ernst et al., ${ }^{7}$ de $12 \times 8 \times 4 \mu \mathrm{m}$.

A Figura 5 apresenta o espectro de refletância difusa na região do infravermelho. A banda a $3600 \mathrm{~cm}^{-1}$ pode ser atribuída à combinação de vibrações simétricas e antissimétricas do $\mathrm{OH}$ da molécula de água ou pode ser devido a vibrações de moléculas de água localizadas em duas posições diferentes na cavidade da zeólita. ${ }^{8}$ A banda a $1630 \mathrm{~cm}^{-1}$ observada refere-se à interação do $\mathrm{OH}$ com o oxigênio da estrutura zeolítica. Entre 300 a $1500 \mathrm{~cm}^{-1}$ observam-se vibrações referentes à 


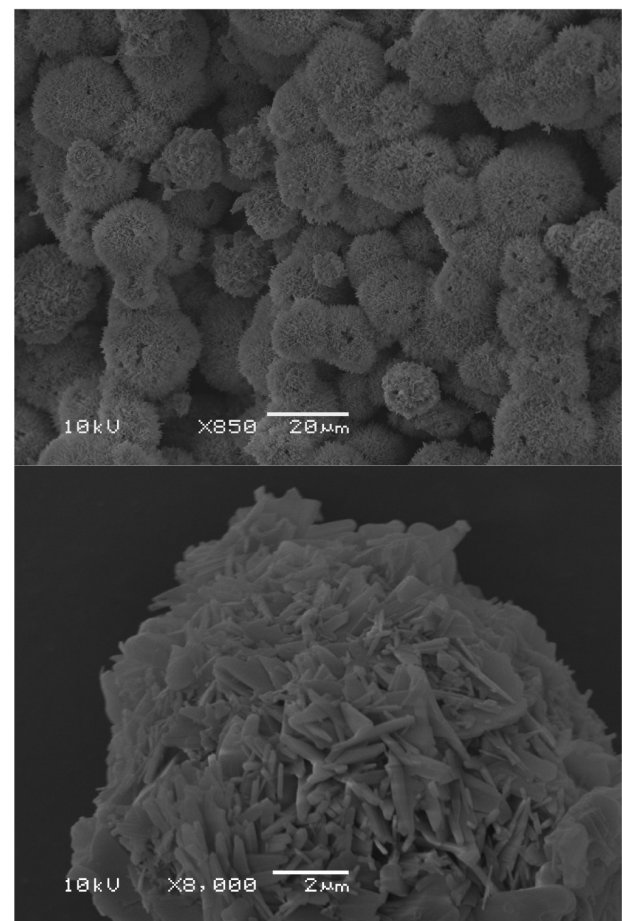

Figura 4. Fotografias de MEV da MCM-71

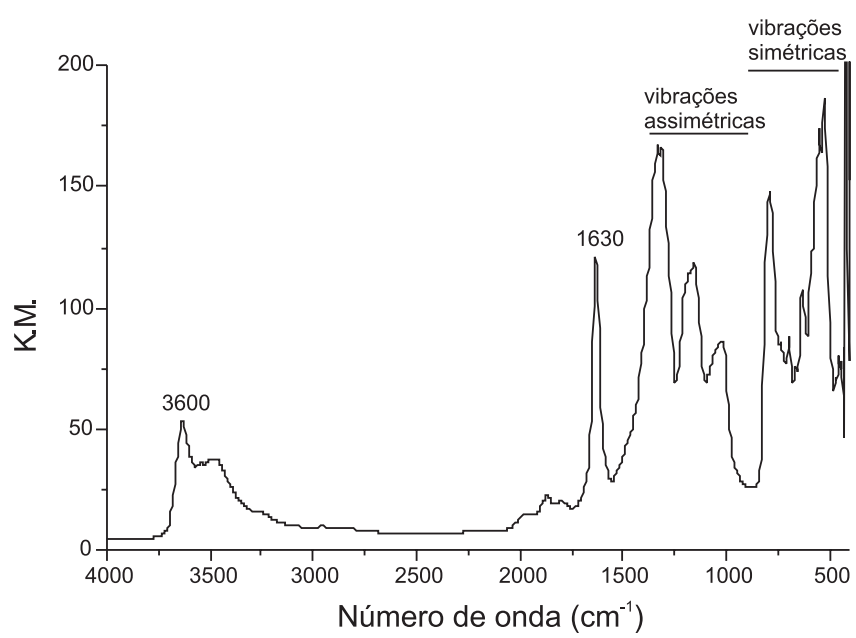

Figura 5. Espectro no infravermelho da amostra MCM-71 estrutura zeolítica. Segundo a correlação $\mathrm{FKS}^{6}$ podem-se classificar as vibrações estruturais e obter informações sobre modificações estruturais. Entretanto, como na literatura não foi apresentado o espectro de infravermelho da MCM-71, fica difícil obter informações da estrutura e observar possíveis modificações. Por trabalhos anteriores em outras estruturas zeolíticas, ${ }^{6}$ sabe-se que vibrações assimétricas ocorrem entre 950 e $1250 \mathrm{~cm}^{-1}$ e vibrações simétricas entre 650 e 950 $\mathrm{cm}^{-1}$. No material MCM-71 sintetizado, observamos a $1030 \mathrm{~cm}^{-1}$ uma vibração assimétrica referente à vibração $\mathrm{TO}_{4}$ insensível à estrutura $\mathrm{e}$ uma outra vibração assimétrica a $1175 \mathrm{~cm}^{-1}$ referente à vibração de ligação de tetraedros $\mathrm{TO}_{4}$ sensíveis à estrutura. Entre 300 e $650 \mathrm{~cm}^{-1}$ observam-se bandas referentes à vibração da estrutura, que no caso da MCM-71 fica difícil de classificar por ser um material novo. Outras estruturas associam estas vibrações a anéis duplos de 6 membros, a aberturas de poros, etc. ${ }^{6}$

\section{CONCLUSÕES}

A zeólita MCM-71 foi sintetizada com sucesso através da síntese hidrotérmica na presença de trietanolamina. Não foi detectada a fase mordenita como impureza, entretanto observa-se quartzo. A zeólita MCM-71 apresentou uma maior acessibilidade quando na forma protônica, sendo possivelmente adequada para o emprego em catálise.

\section{AGRADECIMENTOS}

À URI - Campus Erechim e ao CNPq pelo auxílio financeiro e ao Laboratório de Difração de raios-X - UFRGS pelas análises de DRX.

\section{REFERÊNCIAS}

1. Braga, A. A. C.; Morgon, N. H.; Quim. Nova 2007, 30, 178.

2. Luna, F. J.; Ulf, S.; Quim. Nova 2001, 24, 885.

3. Dhingra, S.; US Pat. 6,645,462 2003.

4. Dhingra, S.; Sherer, E. F.; WO 03/097531 2003.

5. Rigo, R. T.; Luvison, C.; Pergher, S.; Resumos da 30 ${ }^{a}$ Reunião Anual da Sociedade Brasileira de Química, Águas de Lindóia, Brasil, 2007.

6. Giannetto, G.; Montes, A.; Rodríguez, G.; Zeolitas: Características, propiedades y aplicaciones industriales, EdiT: Caracas, 2000.

7. Ernst, S.; Hartmann, M.; Tontisirin, S.; Böhlmann, W.; Stud. Surf. Sci. Catal. 2005, 158, 1287.

8. Hunger, J.; Beta, I. A.; Bolhlig, H.; Ling, C.; Jobic, H.; Hunger, B.; J. Phys. Chem. B 2006, 110, 342. 